IF- 3/95

March 1995

\title{
CHIRAL SYMMETRIES OF THE WZNW MODEL BY HAMILTONIAN METHODS *
}

\author{
B. Sazdović \\ Institute of Physics, P.O.Box 57,11001 Beograd, Yugoslavia
}

\begin{abstract}
The connection between the Kac-Moody algebras of currents and the chiral symmetries of the two dimensional WZNW model is clarified. It is shown that only the zero modes of the Kac-Moody currents are the first class constraints, and that, consequently, the corresponding gauge symmetries are chiral.
\end{abstract}

\footnotetext{
* Work supported in part by the Serbian Reserch Foundation, Yugoslavia.
} 


\section{Introduction}

It is well known that the two dimensional Wess-Zumino-Novikov-Witten (WZNW) model [1-3] in addition to the conformal invariance possesses an invariance under the chiral gauge transformations

$$
g^{\Omega_{+} \Omega_{-}}\left(x^{+}, x^{-}\right)=\Omega_{+}\left(x^{+}\right) g\left(x^{+}, x^{-}\right) \Omega_{-}^{-1}\left(x^{-}\right)
$$

when $\Omega_{+}$and $\Omega_{-}$are arbitrary group valued functions of the light cone coordinates

$$
x^{ \pm}=\frac{1}{\sqrt{2}}\left(x^{0} \pm x^{1}\right) .
$$

It is common in the literature [3-5] to connect the Kac-Moody (KM) currents with the symmetry (1). The goal of this paper is to clarify this connection from the point of view of Dirac's method of constraints [6]. The generators of the symmetry must be the first-class (FC) constraints while "almost all" KM currents are the second-class (SC) constraints. We are going to solve this puzzle by showing that only one Fourier mode of the KM currents is the FC constraint, and this mode is the generator of the chiral symmetry (1). This explains why the symmetry is chiral.

There is another nonstandard application of this canonical analysis. Usualy we use this approach to obtain local gauge symmetries (the parameters of the transformations depend on all space-time coordinates). In this example we are able to find chiral gauge symmetries (the parameters of the transformations depend only on some particular combinations of the coordinates, here $x^{+}$and $x^{-}$). The FC constraints as the generators of these transformations are the Fourier components of the complete constraints.

\section{The two dimensional WZNW model}

We will consider the WZNW model described by the action [3]

$$
S(g)=S_{0}(g)+k \Gamma(g) .
$$

Here

$$
S_{0}(g)=\frac{k}{16 \pi} \int_{\Sigma}\left({ }^{*} v_{-}, v_{-}\right)
$$

is the action of the non linear $\sigma$-model on the field $g: \Sigma \rightarrow G$ where $\Sigma$ is two dimensional space-time and $G$ is a semisimple Lie group. The expression $(X, Y)$ is the Cartan-Killing form, invariant under the adjoint action of the group element $\Omega \in G$

$$
\begin{gathered}
\left(\Omega X \Omega^{-1}, \Omega Y \Omega^{-1}\right)=(X, Y), \\
v_{-}=g^{-1} d g \quad v_{+}=g d g^{-1}=-d g g^{-1}
\end{gathered}
$$


are the left (right) invariant Maurer-Cartan forms and ${ }^{*} v={ }^{*} d x^{\mu} v_{\mu}=\varepsilon^{\mu}{ }_{\nu} d x^{\nu} v_{\mu}$ is the dual of $v$. The second term

$$
\Gamma(g)=\frac{1}{24 \pi} \int_{B} \frac{1}{2}\left(v_{-},\left[v_{-}, v_{-}\right]\right)
$$

is the Wess-Zumino topological term where the integration is carried over a three dimensional manifold $B$, whose boundary is $\Sigma(\Sigma=\partial B)$.

Instead of working with the field $g(x)$ we will benefit from taking local coordinates on the group manifold $\varphi^{\alpha}(x)$ as canonical coordinates. Let $t_{a}$ be the generators of the Lie algebra of $G$ satisfying $\left[t_{a}, t_{b}\right]=f_{a b}{ }^{c} t_{c}$, and let $\gamma_{a b}=\left(t_{a}, t_{b}\right)=f_{a c}{ }^{d} f_{b d}{ }^{c}$ be the group metric. The coordinate indices $\alpha, \beta, \gamma, \ldots$ and the Lie algebra indices $a, b, c, \ldots$ run over the same range.

We define the veilbeins on $G, E_{\alpha}{ }^{a}(\varphi)$, as the coeficients of the expansion of $v$ in $d \varphi^{\alpha}$ and $t_{a}$

$$
v_{A}=d \varphi^{\alpha} E_{A \alpha}^{a}(\varphi) t_{a}=d \varphi^{\alpha} E_{A \alpha}
$$

where $A=+,-$ are light cone indices. There are two veilbeins, $E_{+\alpha}{ }^{a}$ and $E_{-\alpha}{ }^{a}$, as well as two Maurer-Cartan forms, $v_{+}$and $v_{-}$. The metric on the group manifold

$$
H_{\alpha \beta}(\varphi)=\left(E_{A \alpha}, E_{A \beta}\right)=\gamma_{a b} E_{A \alpha}{ }^{a} E_{A \beta}{ }^{b}
$$

(no sumation over A) does not depend on $A\left(H_{+}=H_{-}\right)$as a consequence of the relation $v_{-}=-g^{-1} v_{+} g$ and the property (5) of the Cartan-Killing form.

The action (4a) can be now rewritten in the form

$$
S_{0}(\varphi)=\frac{k}{8 \pi} \int_{\Sigma} d^{2} x\left(\frac{-1}{2}\right) \eta^{\mu \nu} H_{\alpha \beta}(\varphi) \partial_{\mu} \varphi^{\alpha} \partial_{\nu} \varphi^{\beta}
$$

Since the three form $v^{3}$ is a closed form on the three dimensional manifold $B$, we have locally

$$
\frac{1}{2}\left(v_{A},\left[v_{A}, v_{A}\right]\right)=-3 ! d \tau_{A}(\varphi)
$$

where $\tau_{A}$ is a 2 -form

$$
\tau_{A}(\varphi)=\frac{1}{2} \tau_{A \alpha \beta} d \varphi^{\alpha} d \varphi^{\beta}
$$

Note that $\tau_{-}=-\tau_{+}$.Using the Stoke's theorem the Wess-Zumino term $\Gamma$ can be locally converted to an integral over the space time $\Sigma$

$$
\Gamma(\varphi)=-\frac{1}{4 \pi} \int_{\Sigma=\partial B} \tau_{-}=\frac{1}{8 \pi} \int_{\Sigma} d^{2} x \varepsilon^{\mu \nu} \tau_{-\alpha \beta}(\varphi) \partial_{\mu} \varphi^{\alpha} \partial_{\nu} \varphi^{\beta}
$$

The local expression for the action

$$
S(\varphi)=S_{0}(\varphi)+k \Gamma(\varphi)=\kappa \int_{\Sigma} d^{2} x\left[-\frac{1}{2} \eta^{\mu \nu} H_{\alpha \beta}(\varphi)+\varepsilon^{\mu \nu} \tau_{-\alpha \beta}(\varphi)\right] \partial_{\mu} \varphi^{\alpha} \partial_{\nu} \varphi^{\beta}
$$


writen in the light cone coordinates takes the form

$$
S(\varphi)=-\kappa \int_{\Sigma} d^{2} x\left[H_{\alpha \beta}(\varphi)+2 \tau_{-\alpha \beta}(\varphi)\right] \partial_{-} \varphi^{\alpha} \partial_{+} \varphi^{\beta}
$$

where $\kappa \equiv \frac{k}{8 \pi}, \quad \partial_{ \pm}=\frac{1}{\sqrt{2}}\left(\partial_{0} \pm \partial_{1}\right)$ and $\varepsilon^{01}=-1$. The action in the form (3c) is very convenient for the canonical analisys. For the final conclusion we will need boundary conditions, as will be discussed later.

\section{Constraints}

We are going to use Dirac's canonical approach to find the gauge symmetries. The hamiltonian equations of motion describe dinamical evolution of a system by a trajectory in a phase space. The presence of an arbitrary multiplier in the hamiltonian means that for given initial conditions we will not obtain the unique solution, one trajectory, but a whole set of trajectories, describing the same physical state. The FC constraints are generators of the mapping between these trajectories that do not change the phisical state. These unphysical transformations are the gauge symmetries of the hamiltonian equations of motion. The method essentialy depends on the choice of time variable. If we take $x^{0}$ as a time coordinate and use the bilinear expression in the time derivative (3b), as a action, there are no constraints and consequently we can not find the symmetries this way.

The expression $(3 \mathrm{c})$ for the action is more promising because it is linear in the $\partial_{-} \varphi$ and $\partial_{+} \varphi$. Taking first $x^{-}$and then $x^{+}$as time coordinates we will find some primary constraints which give us a hope to find some symmetries of the system. To treat both cases together we will use the light cone indices $A=\{+,-\}$ with $x^{A}=\left\{x^{+}, x^{-}\right\}$and $\partial_{A}=\left\{\partial_{+}, \partial_{-}\right\}$. These two cases are independent and there are no summations over repeated indices $A$.

In the first case $(A=-)$ we will take $x^{-}=\tau$ and $x^{+}=\sigma$ and in the second one $(A=+)$ we will take $x^{+}=\tau$ and $x^{-}=-\sigma$. (The minus sign we adopt to preserve the orientation between coordinate axis.)

The action corresponding to the choice $x^{A}=\tau$ has the form

$$
S_{A}(\varphi)=\kappa \int d^{2} x\left[(-1)^{A} H_{\alpha \beta}-2 \tau_{-\alpha \beta}\right] \dot{\varphi}^{\alpha} \varphi^{\prime \beta} .
$$

The canonical momentum conjugate to the variable $\varphi^{\alpha}$ is

$$
\pi_{\alpha}=\kappa\left[(-1)^{A} H_{\alpha \beta}-2 \tau_{-\alpha \beta}\right] \varphi^{\prime \beta}
$$

and therefore, there are primary constraints:

$$
J_{A \alpha}=\pi_{\alpha}+2 \kappa \tau_{-\alpha \beta} \varphi^{\prime \beta}-(-1)^{A} \kappa H_{\alpha \beta} \varphi^{\prime \beta} .
$$

We prefer to rewrite these constraints with the Lie algebra indices as

$$
J_{A a}=-E_{A a}^{\alpha} J_{A \alpha}
$$


where $E_{A a}{ }^{\alpha}$ is the inverse of $E_{A \alpha}{ }^{a}$. The Poisson brackets (PB) between these constraints define two independent KM algebras $(A=+$ or -$)$ :

$$
\left\{J_{A a}(x), J_{A b}(y)\right\}=f_{a b}^{c} J_{A c}(x) \delta\left(\sigma_{x}-\sigma_{y}\right)-(-1)^{A} 2 \kappa \gamma_{a b} \delta^{\prime}\left(\sigma_{x}-\sigma_{y}\right) .
$$

The expression (14) is a convenient linear combination of the previous constraints. We adapt a correspondence between $J_{ \pm}$and $E_{ \pm}$in order to obtain the algebra (15a).

The last term in the KM algebra contains the derivative of the $\delta$-function and is known as a Schwinger term. In the two dimensional theory with free massless fermions this term originates from the anomalous commutator algebra [3] and has a quantum character. It drastically changes the nature of the constraints [7] from the FC constraints to the SC ones. In the WZNW model the Schwinger term arose classically in the PB algebra, and it has important implications for the nature of the classical symmetries of the theory,

The action is linear in the time derivative and consequently the canonical hamiltonian density is zero, $\mathcal{H}_{c}=0$, while the total hamiltonian takes the form

$$
H_{A T}=\int d \sigma \mathcal{H}_{A T}=\int d \sigma u_{A}^{a} J_{A a}
$$

The consistency condition for $J_{A a}$ leads to

$$
\dot{J}_{A a}(x)=\left\{J_{A a}(x), H_{A T}\right\} \approx-(-1)^{A} 2 \kappa u_{A a}^{\prime}(x) \approx 0 .
$$

The multipliers in (17a) looks like determined $u_{A a}^{\prime}=0$ which means that $J_{A a}$ should be SC constraints. More precisely, only the first derivative of the multipliers is determined and the corresponding constraints are the SC. The $\sigma$-independent part is arbitrary function of $\tau$ and corresponding constraints are the FC. The same conclusion we can obtain from eq. (15a). The Schwinger term contains the derivative of the $\delta$-function and gives zero on the $\sigma$-independent functions. It means that the Schwinger term does not give a contributions for some modes of the currents and these modes are the FC constraints.

Let us make a more precise investigation. We assume that space is compact which means that for all variables $X=\left\{J_{A a}, u_{A}{ }^{a}, \ldots\right\}$ we have $X(\tau, \sigma+L)=X(\tau, \sigma)$. We will use the Fourier expansion

$$
X(x)=\frac{1}{L} \sum_{n \in Z} X_{n}\left(\tau_{x}\right) e^{-i n \omega \sigma_{x}}, \quad X_{n}\left(\tau_{x}\right)=\int_{0}^{L} d \sigma_{x} X(x) e^{i n \omega \sigma_{x}}
$$

where $\omega=\frac{2 \pi}{L}$, and the $\delta$-function representation

$$
\delta(\sigma)=\frac{1}{L} \sum_{n \in Z} e^{-i n \omega \sigma}
$$

The PB between modes $J_{\text {Aan }}(\tau)$ are

$$
\left\{J_{A a n}, J_{A b m}\right\}=f_{a b}^{c} J_{A c(n+m)}-(-1)^{A} 4 \pi i \kappa n \gamma_{a b} \delta_{n+m, 0}
$$


while plus and minus constraints commute. This expression does not depend on the period $L$. In terms of modes the total hamiltonian becomes

$$
H_{A T}\left(\tau_{x}\right)=\int_{0}^{L} d \sigma_{x} \mathcal{H}_{A T}(x)=\frac{1}{L} \sum_{n \in Z} u_{A}{ }^{a}{ }_{n}\left(\tau_{x}\right) J_{A a(-n)}\left(\tau_{x}\right) .
$$

Now it is easy to separate the FC and the SC constraints, and check the consistency condition for the primary constraints $J_{\text {Aan }}(\tau)$ :

$$
\dot{J}_{\text {Aan }}(\tau)=\left\{J_{A a n}(\tau), H_{A T}\right\} \approx-(-1)^{A} \frac{4 \pi i}{L} \kappa n u_{A a n}(\tau) \approx 0
$$

For $n=0$ the multipliers $u_{A a 0}(\tau)$ are arbitrary functions of time and $J_{A a 0}(\tau)$ constraints are FC. For $n \neq 0$ we can determined the multipliers $u_{\text {Aan }}(\tau)=0(n \neq 0)$ which means that $J_{\text {Aan }}(n \neq 0)$ are SC constraints. The same conclusion follows from (15b) because

$$
\begin{aligned}
& \left\{J_{A a 0}, J_{A b n}\right\}=f_{a b}^{c} J_{A c n} \approx 0 \\
& \left\{J_{A a n}, J_{A b(-n)}\right\} \approx-(-1)^{A} 4 \pi i \kappa n \gamma_{a b} \neq 0 \quad \text { for } \quad n \neq 0 .
\end{aligned}
$$

In any case there are no secondary constraints. The total hamiltonian is now

$$
H_{A T}=\frac{1}{L} u_{A}{ }_{0}(\tau) J_{A a 0}(\tau)
$$

In sec.5 we will verify that expression (16c) gives the correct equations of motion.

The assumption of the periodicaly boundary conditions is not necessary. The general solution of (17a) is $u_{A a}(\sigma, \tau)=u_{A a}(\tau)$ and substituting this into (16a) we obtain (16c) with $u_{A}{ }^{a}(\tau)$ instead of $\frac{1}{L} u_{A}{ }^{a}{ }_{0}(\tau)$ and $J_{A a 0}=\int d \sigma J_{A a}(\sigma, \tau)$. The constraints $J_{A a 0}$ are FC because they correspond to the arbitrary multiplier $u_{A a}(\tau)$. They also weakly vanish with all constraints, which can be easily verified integrating eq.(15a) over $\sigma_{x}$. For our purpose any boundary condition with finite $J_{A a 0}$ is a good condition.

\section{Chiral gauge symmetries}

The presence of arbitrary multipliers $u_{A}{ }^{a}{ }_{0}(\tau)$ in the total hamiltonian means that the theory has a gauge symmetry. We will use Castellani's method [8] to obtain the gauge generators and the corresponding gauge transformations. The gauge parameters $\omega_{A}{ }^{a}(\tau)$ will depend only on the time coordinate $\tau$, as well as the $\mathrm{FC}$ constraints $J_{A a 0}$ and the arbitrary multipliers $u_{A}{ }^{a}$.

All the FC constraints $J_{A a 0}$ are primary in this case, and the expression for the gauge symmetry generator is

$$
G_{A}=\omega_{A}^{a}(\tau) J_{A a 0}(\tau) . \quad(A=+,-)
$$


The gauge transformations produced by $G_{A}$ are

$$
\delta_{A} \varphi^{\alpha}=\left\{\varphi^{\alpha}, G_{A}\right\}=-\omega_{A}^{a}(\tau) E_{A a}{ }^{\alpha} .
$$

We are interested in the gauge transformations of the field $g$. Using eqs.(6) and (8) we can connect the transformations of the fields $g$ and $\varphi$

$$
g^{-1} \delta_{-} g=\delta_{-} \varphi^{\alpha} E_{-\alpha}{ }^{a} t_{a} \quad-\delta_{+} g g^{-1}=\delta_{+} \varphi^{\alpha} E_{+\alpha}{ }^{a} t_{a} .
$$

With the help of eq.(22) we get

$$
\delta_{-} g=-g \omega_{-} \quad \delta_{+} g=\omega_{+} g
$$

where $\omega_{A}=\omega_{A}{ }^{a} t_{a}$.

Because $\omega_{A}$ is infinitesimal we can write

$$
g^{\omega_{-}}=g+\delta_{-} g=g\left(1-\omega_{-}\right) \approx g e^{-\omega_{-}} \quad g^{\omega_{+}}=g+\delta_{+} g=\left(1+\omega_{+}\right) g \approx e^{\omega_{+}} g .
$$

Recalling that $\omega_{-} \equiv \omega_{-}\left(x^{-}\right)$and $\omega_{+} \equiv \omega_{+}\left(x^{+}\right)$we have

$$
g^{\Omega_{-}}=g \Omega_{-}^{-1}\left(x^{-}\right) \quad g^{\Omega_{+}}=\Omega_{+}\left(x^{+}\right) g
$$

where $\Omega_{A}\left(x^{A}\right)=e^{\omega_{A}\left(x^{A}\right)}$. Equations (26) are equivalent with the equation (1). Therefore we demonstrated that the gauge symmetries generated by the zero modes of the KM currents are exactly the known chiral gauge symmetries.

\section{Equations of motion}

For completeness we briefly derive the hamiltonian equations of motion. For the fields $\varphi^{\alpha}$ we have

$$
\dot{\varphi}^{\alpha}=\left\{\varphi^{\alpha}, H_{A T}\right\}=-u_{A}{ }^{a}{ }_{0} E_{A a}{ }^{\alpha} .
$$

The equations of motion for the field $g$ are obtained from eqs.(6)and (8) in terms of the equations for $\varphi^{\alpha}$

$$
g^{-1} \dot{g}=\dot{\varphi}^{\alpha} E_{-\alpha}{ }^{a} t_{a} \quad-\dot{g} g^{-1}=\dot{\varphi}^{\alpha} E_{+\alpha}{ }^{a} t_{a}
$$

and by using eq.(27) we find

$$
g^{-1} \dot{g}=-u_{-0}(\tau) \quad \dot{g} g^{-1}=u_{+0}(\tau)
$$

where $u_{A 0}=u_{A}{ }^{a} t_{a}$.

If we now recall our definition of time $\tau=x^{A}$, it follows that $\dot{g}=\partial_{-} g$ for $A=-$ and $\dot{g}=\partial_{+} g$ for $A=+$, so that eqs.(29a) can be rewritten as

$$
g^{-1} \partial_{-} g=-u_{-0}\left(x^{-}\right) \quad \partial_{+} g g^{-1}=u_{+0}\left(x^{+}\right) .
$$

Therefore we obtain the well known equations of motion,

$$
\partial_{+}\left(g^{-1} \partial_{-} g\right)=0 \quad \partial_{-}\left(\partial_{+} g g^{-1}\right)=0
$$

which are, as well as the equations (29b), evidently invariant under the chiral gauge transformations (1). 


\section{Conclusion}

The canonical formalism in the light cone coordinates is developed for the two dimensional WZNW model. Using $x^{-}$and $x^{+}$as the evolution parameters we obtain the two commuting $\mathrm{KM}$ algebras of constraints. We made a mode expansion in order to separate the FC constraints from the SC ones. Only the zero modes are FC constraints and they are the generators of the gauge symmetries. The only arbitrary parameters are zero mode gauge parameters. They depend on the $x^{-}$in the first case and on the $x^{+}$in the second one, and this explains the chirality of the gauge symmetry.

The connection between KM algebra and chiral gauge symmetry is now clear. The KM algebra contains infinitely many modes of the SC constraints (it looks like the algebra of SC constraints) and only one FC mode which is the generator of the symmerty (1).

This result is obtained by the application of the canonical approach to a new kind of symmetry when the gauge parameter does not depend on all coordinates, but only on some particular combinations of the coordinates [9].

\section{References}

[1] J. Wess and B. Zumino, Phys.Lett. 37B (1971) 95.

[2] S. P. Novikov, Sov.Math.Doklady 24 (1981) 222; Usp.Math.Nauk. 37 (1982) 3.

[3] E. Witten, Nucl.Phys. B223 (1983) 422; Commun.Math,Phys. 92 (1984) 455.

[4] V. G. Knizhnik and A. B. Zamolodchikov, Nucl.Phys. B247 (1984) 83.

[5] D. Gepner and E. Witten, Nucl.Phys. B278 (1986) 493.

[6] P. A. M. Dirac, Lectures on quantum mechanics, Belfer Graduate School of Science (Yeshiva Univ., 1964).

[7] L. D. Faddeev, Phys.Lett. 145B (1984) 81;

L. D. Faddeev and S. L. Shatashvili, Phys.Lett. 167B (1986) 225.

[8] L. Castellani, Ann.Phys. (New York) 143 (1982) 357.

[9] M. Blagojević, M. Vasilić and T. Vukašinac, Class.Quan.Grav. 11 (1994) 2134. 